\title{
Modeling of Non-Linear and Hysteretic Magnetization Effects in Transient Potential Drop Measurements
}

\author{
Øyvind PERSVIK ${ }^{1}$ and Zhiliang ZHANG \\ Department of Structural Engineering, Norwegian University of Science and \\ Technology (NTNU), 7491 Trondheim, Norway
}

\begin{abstract}
We have compared transient potential drop measurements on ferromagnetic steel rods with finite difference simulation assuming that the magnetization varies as a quadratic function of the applied field. Good agreement between simulation and experimental measurement is achieved and the results are discussed in terms of the Rayleigh law of magnetization.
\end{abstract}

Keywords. transient, potential drop, skin effect, non-linear magnetization, Rayleigh law

\section{Introduction}

Modeling of the response of electromagnetic sensors is a key step in their use for structural health monitoring (SHM) and the evaluation of material properties of ferromagnetic materials such as carbon steel. Commonly, non-linearity in magnetic properties is ignored due to the expediency of assuming linear properties. On the other hand, understanding the implications of non-linear effects can be important when interpreting data. Additionally, non-linear and hysteretic effects are characteristic features of ferromagnetic materials, related to irreversible motion of domain walls in the presence of material defects [1], which may be exploited in non-destructive evaluation (NDE). An example of this principle is the magnetic barkhausen noise (MBN) method [2] where the measured response is due to discontinuous magnetization jumps.

Here we consider the transient potential drop method $[3,4]$ where, by using a pulsed current as the source, sensitivity to varying depths is achieved rapidly and by experimentally simple means. Due to the skin effect the variation of material properties and geometrical features with depth is manifested as a time dependent variation in the transient potential drop analogously to the frequency dependence in alternating current potential drop (ACPD) [5]. Furthermore, recording the transient potential drop due to a pulsed current is analogous to the use of pulsed current in eddy current testing.

The Rayleigh law of magnetization is commonly used as a simple model for the magnetization of ferromagnetic materials at low fields [6]; by including a quadratic term in the field dependence it can be viewed as a first approximation to non-linear magneti-

\footnotetext{
${ }^{1}$ Corresponding Author: Øyvind Persvik; E-mail: oyvind.persvik@ntnu.no.
} 
zation. In a previous similar work [7] ACPD was used to measure the parameters in the Rayleigh law by using the finite difference method to obtain the steady state response due to a sinusoidal drive current. Also, non-constant permeability in steel has been studied in a different approach by interpreting the results of four-point ACPD measurements on steel plates using a linear model and a frequency dependent complex relative permeability [8].

In this paper we report on initial results from modeling of non-linear and hysteretic effects in transient potential drop measurements on ferromagnetic steel. Our approach is similar to that described in [7], notably by using cylindrical rod samples and finite difference simulation, but our main motivation is to compare transient measurements with numerical simulation in the time domain directly. Firstly, this is to validate the approach for predicting transient signals due to pulsed currents when models assuming constant permeability are inadequate. Secondly we discuss the results in view of the Rayleigh law by comparing the reversible and hysteretic responses to unipolar drive current.

\section{Transient Potential Drop}

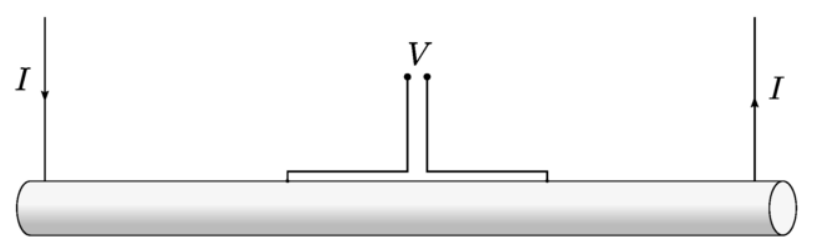

Figure 1. Transient potential drop measurement on a metal rod.

In the transient potential drop method the time domain response due to a pulsed current is measured. Assuming a homogeneous conducting rod (Figure 1), with radius $a$, conductivity $\sigma$ and constant relative permeability $\mu_{r}$, the response to an injected step-like current with exponential rise time $\tau, I(t)=I_{0}\left(1-e^{-t / \tau}\right)$, is given by [9]

$$
V(t)=V_{0}\left(1-e^{-t / \tau}+\sum_{n=1}^{\infty} \frac{e^{-t / \kappa_{n}}-e^{-t / \tau}}{1-\tau / \kappa_{n}}\right) .
$$

Here $V(t)$ is the voltage measured between contact probes on the surface separated by a distance $l, V_{0}$ is the response in the direct current (DC) limit, $V_{0}=l / \pi \sigma a^{2}$, and $\kappa_{n}=$ $\mu \sigma a^{2} / \beta_{n}^{2}$ where $\beta_{n}$ is the $n$-th zero of the Bessel function of the first kind of order 1 . The parameter $\kappa=\mu \sigma a^{2}$ is a characteristic decay time of the transient.

Figure 2 shows an example response to a current pulse of width $T$ with exponentially rising and falling edges. The skin-effect voltage represents the summation term in Eq. (1) and is the result of induced eddy currents due to the time-varying injected current.

\subsection{Finite difference simulation}

In time dependent potential drop problems, quasi-static electromagnetic fields due to a time-varying current source are obtained from Maxwell's equations and are used to cal- 


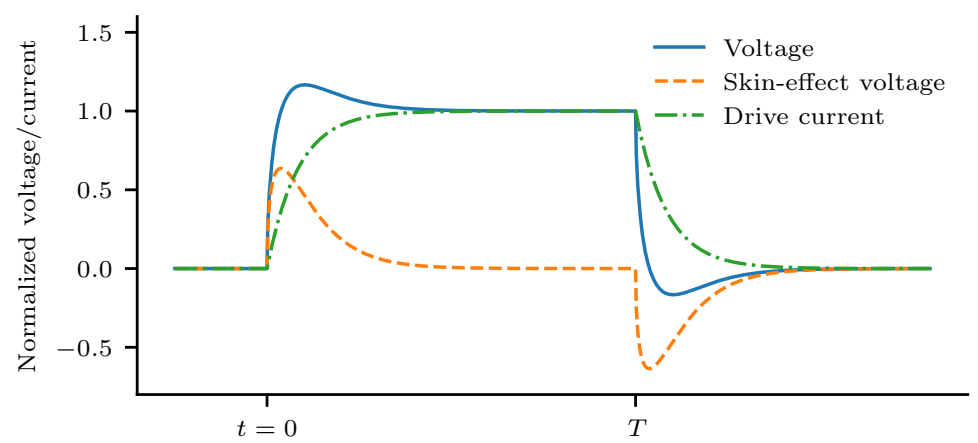

Figure 2. Example of the transient response to a pulsed drive current with rise time $\tau=\kappa / 10$ and $T=1.2 \kappa$.

culate the surface potential. An axially symmetric field $\mathbf{H}(\rho, t)=H(\rho, t) \hat{\boldsymbol{\phi}}$ in a cylindrical rod is governed by

$$
\nabla^{2} H-\frac{H}{\rho^{2}}=\mu_{0} \sigma\left(1+\frac{\mathrm{d} M}{\mathrm{~d} H}\right) \frac{\partial H}{\partial t} .
$$

Here $M$ is the magnetization in the material and the right hand side corresponds to the rate of change of the magnetic flux density $\partial B / \partial t$ in Faraday's law.

A first approximation to non-linear magnetization is to assume that the magnetization varies with field strength according to $\mathrm{d} M / \mathrm{d} H=\alpha+\beta H$. To determine the transient potential drop numerically a finite difference scheme is formulated in terms of normalized variables $u=H / \Delta H, x=\rho / a$, and $\bar{t}=t / \kappa_{\alpha}$ with $\kappa_{\alpha}=(1+\alpha) \sigma a^{2}$. This gives the non-dimensional equation

$$
\nabla^{2} u-u=(1+q u) \frac{\partial u}{\partial \bar{t}}
$$

where the parameter $q=\beta \Delta H /(1+\alpha)$ reflects the (non-linear) field dependence. For simplicity an explicit scheme is implemented numerically. In Figure 3 a simulated transient is compared with the exact formula, Eq. (1), verifying that the numerical results converge to the expected transient solution in the case of constant $\mu_{r}$.

The form of $\mathrm{d} M / \mathrm{d} H$ gives a quadratic field dependence corresponding to the Rayleigh law describing the initial magnetization at low fields by $M_{i}=\chi_{i} H+\eta H^{2}$, with initial susceptibility $\chi_{i}$ and the Rayleigh constant $\eta$, and the minor hysteresis loop traced out by a field that varies between $\pm H_{0}$ by $M_{h}=\left(\chi_{i}+\eta H_{0}\right) H \pm \frac{\eta}{2}\left(H_{0}^{2}-H^{2}\right)$.

In the case of unipolar current excitation, typical in pulsed measurements, the magnetic field varies between 0 and $H_{0}=I_{0} / 2 \pi a$ on the surface and its maximal value decreases linearly with the radial distance to the center of the rod where it is zero in accordance with Ampere's law. The initial response from a demagnetized state follows $d M_{i} / d H=\chi_{i}+2 \eta H$ and induces both reversible and irreversible magnetization (indicated in Figure 4) whereas the reversible response to subsequent pulses is assumed to follow $d M_{r} / d H=\chi_{i}+\eta H$. In other words the difference between the initial (hysteretic) response and the reversible response is a factor of 2 in the non-linear parameter. This reflects that one half of the quadratic term in the initial magnetization curve is attributed to 


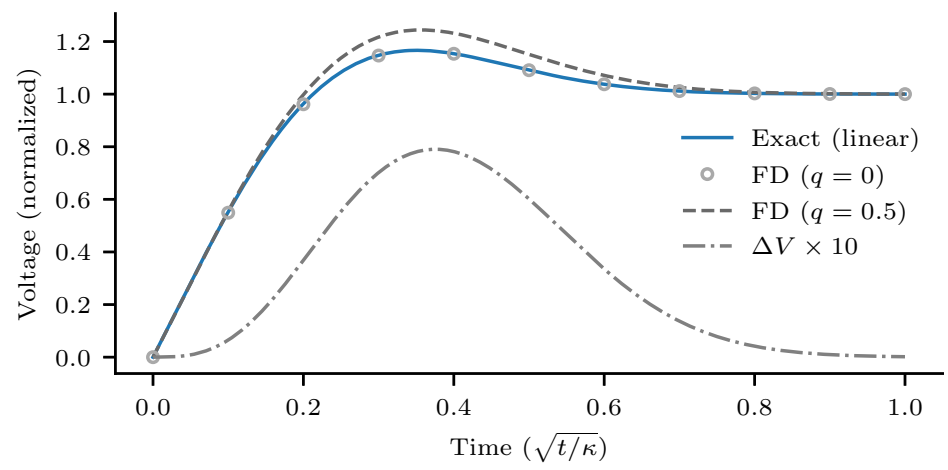

Figure 3. Comparison between theory, Eq. (1), and finite difference simulation $\left(N_{z}=500\right)$ assuming constant $\mu_{r}(q=0)$ and rise time $\tau=\kappa / 10$. Also shown is the simulation result for $q=0.5$ indicating the effect of non-linear magnetization.

irreversible magnetization. This is considered in the following as a guide to interpreting the experimental measurements in terms of the Rayleigh law.

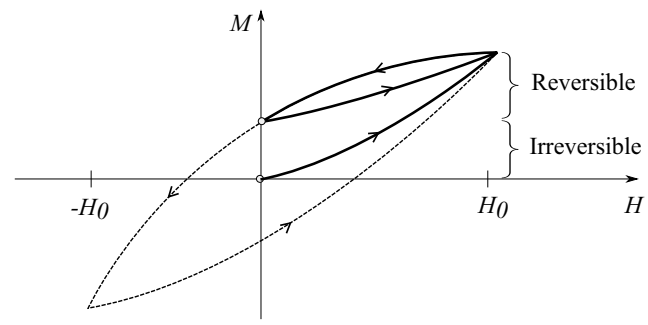

Figure 4. Schematic illustration of minor hysteresis loops. The outer loop is traced out by a field varying between $\pm H_{0}$. The center point $M_{0}$ corresponds to an initial state that may be reached by a demagnetizing cycle where the applied field is reduced gradually to zero.

\section{Experiment}

The experimental setup consists of an arbitrary waveform generator (AWG), a drive current amplifier, and a PC oscilloscope to record the waveforms of the transient potential drop and the drive current.

Measurements were made on a cylindrical rod sample made of structural steel (grade S235), having a nominal diameter $2 a=10 \mathrm{~mm}$ and length of $150 \mathrm{~mm}$. The sample was initially demagnetized in a solenoid. The current was injected by clamps mounted at the ends and the potential drop was measured using spring-loaded pins separated by a distance $l=70.6 \mathrm{~mm}$. In a typical measurement the AWG is programmed with a pulse that rises and falls exponentially. By triggering the AWG the response to a single pulse, or several in succession, is recorded. To achieve a magnetization state corresponding to the center of the minor hysteresis loop for a given magnitude of the drive current (see Figure 4), a low frequency sinusoidal current was injected into the sample with a 
magnitude reduced from $1 \mathrm{~A}$ to zero over several cycles. The drive current in the potential drop measurements has a rise time of $2 \mathrm{~ms}$ and an amplitude of $0.8 \mathrm{~A}\left(H_{0} \approx 25 \mathrm{~A} / \mathrm{m}\right)$.

\section{Results and Discussion}

Figure 5 shows the results of fitting the numerical model to the measurements of the initial response after demagnetization and the reversible response. The model parameters $\kappa$ and $q$ were estimated by non-linear least squares optimization. The differences between the measured transients and the predicted linear responses are shown to better visually discern the relatively small variation due to non-linear effects. Good agreement between the model calculations and measurements is achieved.
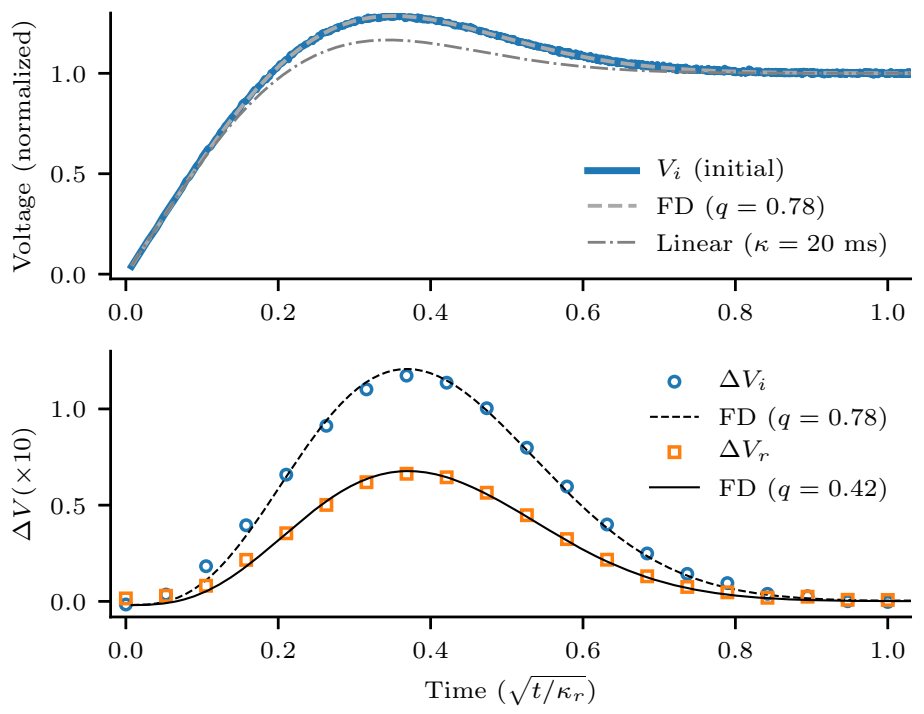

Figure 5. Comparison between measurements and finite difference simulation. The transient response $V_{i}$ (top) corresponds to the initial response after demagnetization. The estimated non-linear contribution $\Delta V_{i}$ to the initial response is greater than $\Delta V_{r}$ (reversible response) due to irreversible magnetization.

Values of the parameters are $\kappa_{i}=20.0 \mathrm{~ms}, q_{i}=0.78$ for the initial response and $\kappa_{r}=20.7 \mathrm{~ms}, q_{r}=0.42$ for the reversible response. In terms of the Rayleigh law, the corresponding values of the relative permeability and the Rayleigh constant $\eta$ are 103 and 1.59 for the initial response and 107 and 1.76 for the reversible response. The value of $q_{i}$ is larger than $q_{r}$ as expected due to the contribution of irreversible magnetization in the initial response. The ratio $q_{i} / q_{r}=1.88$ is less than the factor 2 predicted by the Rayleigh law. Consequently there is a $10 \%$ difference in the values of $\eta$ and there is also a $4 \%$ difference in $\mu_{r}$ whereas ideally both parameters would be treated as constants in the Rayleigh regime. In comparison, the standard errors in the parameter estimates are less than $1 \%$ meaning the variation is not caused by random measurement error alone. Additional uncertainty derive from potential inhomogeneity of the sample, which could bias the fit results. Also, lacking an independent direct measurement, there is some uncertainty in the accuracy of the Rayleigh law itself for the material. 
Table 1 gives some preliminary data for additional samples, obtained as part of a study to quantify effects of treatments such as annealing and work hardening, processes that modify the magnetic properties by changes in stress and microstructure [1]. Clearly, these treatments greatly influence both the linear and non-linear parameters. Indeed, numerous factors are known to affect the magnetic properties of ferromagnetic materials which poses both opportunities and challenges in NDE applications. In this regard the analysis of the non-linearity in the response could provide additional information as an aid in the interpretation of data. In particular the relationship between plastic deformation and the Rayleigh parameters measured using the approach outlined here is the subject of future work.

\begin{tabular}{lllll}
\hline Sample & $\sigma(\mathrm{MS} / \mathrm{m})$ & $\mu_{r}$ & $\eta$ & $\eta / \mu_{r}(\times 100)$ \\
\hline S235 (cold drawn) & 6.1 & 107 & 1.76 & 1.6 \\
S235 (annealed) & 6.1 & 237 & 2.7 & 1.1 \\
S355 & 4.5 & 133 & 1.15 & 0.8 \\
S355 (deformed) & 4.5 & 110 & 0.47 & 0.4 \\
\hline
\end{tabular}

Table 1. Results for different samples. The deformed S355 sample (yield strength $355 \mathrm{MPa}$ ) was plastically deformed in uniaxial tension to $5 \%$ strain.

\section{Conclusions}

We have modeled non-linear effects in transient potential drop measurements using finite difference simulation assuming axial symmetry and a quadratic field dependence of the magnetization. The numerical results are in good agreement with experimental measurements on carbon steel rods. Here we have initially focused on modeling of the response and interpreting the results in view of the Rayleigh law. The approach may prove useful in interpretation of NDE data by providing additional information compared to an analysis of the relative permeability alone.

\section{References}

[1] B Cullity and C Graham. Introduction to Magnetic Materials. John Wiley \& Sons, Inc., 2 edition, 2009.

[2] D C Jiles. Review of magnetic methods for nondestructive evaluation. NDT Int., 21(5):311-319, 1988.

[3] $\varnothing$ Persvik and J R Bowler. Evaluation of four-point transient potential drop on conductive plates. Appl. Phys. Lett., 110(8):084102, 2017.

[4] H Hognestad and A Honne. Determination of stress in ferromagnetic steel by potential drop measurements. Mater. Sci. Tech., 14(11):1109-1114, 1998.

[5] J R Bowler, Y Huang, H Sun, J Brown, and N Bowler. Alternating current potential-drop measurement of the depth of case hardening in steel rods. Meas. Sci. Technol., 19(7):075204, 2008.

[6] D C Jiles. Introduction to magnetism and magnetic materials. CRC Press, 1998.

[7] J I Etcheverry and G A Sánchez. Simple measurement of electromagnetic properties of steel in the rayleigh domain. AIP Conf. Proc., 1511(1):1174-1179, 2013.

[8] N Bowler. Frequency-dependence of relative permeability in steel. AIP Conf. Proc., 820, 2006.

[9] H E Knoepfel. Magnetic Fields: A Comprehensive Theoretical Treatise for Practical Use. John Wiley \& Sons, 2000. 